\title{
To PSG or not to PSG: That is the question
}
A. Ioana Cristea ${ }^{1}$, Christopher D. Baker ${ }^{2}$.

1. Indiana University School of Medicine, Indianapolis, Indiana.

2. Pediatric Heart Lung Center, University of Colorado School of Medicine, Aurora, Colorado.

Keywords: Bronchopulmonary Dysplasia (BPD) Mechanical Ventilation Respiratory Technology Sleep Medicine

This is the author's manuscript of the article published in final edited form as:

Cristea, A. I., \& Baker, C. D. (2020). To PSG or not to PSG: That is the question. Pediatric Pulmonology, 55(1), 910. https://doi.org/10.1002/ppul.24515 
Although once considered only in the most severe cases where mortality was quite likely, tracheostomy placement for long-term chronic ventilation has become more commonplace for infants with severe ventilator-dependent bronchopulmonary dysplasia (BPD) who fail attempts to wean positive pressure ventilation after reaching term gestation.1 As many of these children will be liberated from long-term ventilation in early childhood, tracheostomy decannulation becomes possible. Whether or not to evaluate these children by polysomnography (PSG) before tracheostomy decannulation has been a subject of much debate. 2

In this issue of Pediatric Pulmonology, Quinlan et al3 describe the experience of one institution with respect to tracheostomy decannulation of children with severe BPD. They performed a retrospective chart review of children with tracheostomy/ventilatordependent severe BPD who were ready for decannulation over an approximately 10-year period. Their aim was to determine whether tracheostomy-dependent children with BPD undergoing decannulation who had predecannulation PSG had a higher decannulation success rate than those without predecannulation PSG. One hundred twenty-five tracheostomy-dependent patients with BPD were identified who were considered for decannulation. Forty-six (37\%) underwent PSG and, of those, 19 did not undergo decannulation within the 6-month period after the study. Decannulation failure rates were $3 \%$ when PSG was performed and $5 \%$ when it was not. The authors conclude that decannulation can be successful without PSG in some patients, but that PSG is valuable for identifying which patients with BPD may benefit from treatment of obstructive sleep apnea before decannulation.

The most important finding in this study pertains to the 19 subjects with abnormal PSG who might otherwise have suffered from an unsuccessful decannulation. However, there is an important population that could not be studied due to the retrospective study design. Namely, the authors were not able to identify those patients with tracheostomy-dependent BPD who did not undergo PSG and yet were deemed unsuitable for a decannulation trial based on clinical judgement. Indeed, only an inclusive trial of all tracheostomy-dependent children with BPD would permit comparison of those who were not decannulated whether or not PSG was performed. 
This paper adds to the body of evidence suggesting that decannulation is a complex multistep process that can be done safely once a child's respiratory condition has improved. In 2016, Cristea et al,4 Henningfeld et al,5 and Liptzin et al6 described the experiences of three institutions with respect to weaning mechanical ventilation and tracheostomy decannulation. Whether in the sleep lab using PSG or not, all groups agreed that close monitoring is necessary to determine readiness for tracheostomy decannulation. Importantly, evaluation of the airway (including the glottis and subglottis) by either flexible or rigid bronchoscopy was recommended in all cases.

Tracheostomy decannulation is a shared goal of patients, families, and providers. Despite its clear benefit in many cases, tracheostomy is associated with increased morbidity and mortality.7 Determining the safety of tracheostomy decannulation in children is difficult and carries an element of risk. Evaluation by PSG in a sleep center with pediatric expertise before decannulation can provide valuable information about patient readiness as well as the need for surgical intervention before decannulation. However, these centers are not available at all medical centers in the United States. In addition, evaluation in a sleep center is costly and may delay care.

In conclusion, there are no universally agreed-upon protocols for tracheostomy decannulation. This descriptive study highlights again the importance of and need for multicenter, prospective studies to evaluate tracheostomy decannulation in patients with and without BPD. 


\section{REFERENCES}

1 Baker, C. D. (2019). Long-term ventilation for children with chronic lung disease of infancy. Current Opinion in Pediatrics, 31(3), 357-366. https://doi.org/10.1097/MOP.0000000000000757

2 Cristea, A. I., \& Baker, C. D. (2016). Ventilator Weaning and Tracheostomy Decannulation in Children: More Than One Way. Pediatric Pulmonology, 51(8), 773-774. https://doi.org/10.1002/ppul.23418

3 Quinlan, C., Piccione, J., Kim, J.-Y., Beck, S. E., Brooks, L., Chandy-Patel, R., Escobar, E., \& Afolabi-Brown, O. (2019). The role of polysomnography in tracheostomy decannulation of children with bronchopulmonary dysplasia. Pediatric Pulmonology, 54(11), 1676-1683. https://doi.org/10.1002/ppul.24474

4 Cristea, A. I., Jalou, H. E., Givan, D. C., Davis, S. D., Slaven, J. E., \& Ackerman, V. L. (2016). Use of polysomnography to assess safe decannulation in children. Pediatric Pulmonology, 51(8), 796-802. https://doi.org/10.1002/ppul.23395

5 Henningfeld, J. K., Maletta, K., Ren, B., Richards, K. L., Wegner, C., \& D'Andrea, L. A. (2016). Liberation from home mechanical ventilation and decannulation in children. Pediatric Pulmonology, 51(8), 838-849. https://doi.org/10.1002/ppul.23396

6 Liptzin, D. R., Connell, E. A., Marable, J., Marks, J., Thrasher, J., \& Baker, C. D. (2016). Weaning nocturnal ventilation and decannulation in a pediatric ventilator care program. Pediatric Pulmonology, 51(8), 825-829. https://doi.org/10.1002/ppul.23436

7 DeMauro, S. B., D’Agostino, J. A., Bann, C., Bernbaum, J., Gerdes, M., Bell, E. F., Carlo, W. A., D’Angio, C. T., Das, A., Higgins, R., Hintz, S. R., Laptook, A. R., Natarajan, G., Nelin, L., Poindexter, B. B., Sanchez, P. J., Shankaran, S., Stoll, B. J., Truog, W., ... Kirpalani, H. (2014). Developmental Outcomes of Very Preterm Infants with Tracheostomies. The Journal of Pediatrics, 164(6), 1303-1310.e2. https://doi.org/10.1016/j.jpeds.2013.12.014 